\title{
The usefulness of cardiac CT integrated with FFRCT for planning myocardial revascularization in complex coronary artery disease: a lesson from SYNTAX studies
}

\author{
Daniele Andreini ${ }^{1,2}$, Saima Mushtaq ${ }^{1}$, Edoardo Conte ${ }^{1}$, Mariachiara Mei $^{3}$, Flavia Nicoli ${ }^{1}$, \\ Eleonora Melotti ${ }^{1}$, Giulio Pompilio ${ }^{1,2}$, Mauro Pepi ${ }^{1}$, Antonio L. Bartorelli ${ }^{1,4}$, Yoshinobu Onuma ${ }^{5}$, \\ Patrick W. Serruys ${ }^{6}$
}

${ }^{1}$ Monzino Cardiology Center, IRCCS, Milan, Italy; ${ }^{2}$ Department of Clinical Sciences and Community Health, Cardiovascular Section, University of Milan, Milan, Italy; ${ }^{3}$ Sapienza University, Policlinico Umberto I, Rome, Italy; ${ }^{4}$ Department of Biomedical and Clinical Sciences "Luigi Sacco", University of Milan, Milan, Italy; ${ }^{5}$ Thoraxcenter, Erasmus MC, The Netherlands; ${ }^{6}$ Department of Cardiology, Royal Brompton and Harefield Hospitals, Imperial College London, London, UK

Contributions: (I) Conception and design: D Andreini, S Mushtaq, G Pompilio, AL Bartorelli, Y Onuma, PW Serruys; (II) Administrative support: E Conte, M Mei, F Nicoli, E Melotti, M Pepi; (III) Provision of study material or patients: All authors; (IV) Collection and assembly of data: D Andreini, S Mushtaq, E Conte, M Mei, F Nicoli, E Melotti, Y Onuma, PW Serruys; (V) Data analysis and interpretation: D Andreini, S Mushtaq, M Pepi, Y Onuma, PW Serruys; (VI) Manuscript writing: All authors; (VII) Final approval of manuscript: All authors.

Correspondence to: Daniele Andreini, MD, PhD, FESC, FSCCT. Via C. Parea 4, 20138 Milan, Italy. Email: daniele.andreini@ccfm.it.

\begin{abstract}
After two decades of clinical use, during which coronary CT angiography (CCTA) was considered an appropriate method for the non-invasive assessment of patients with suspected stable coronary artery disease (CAD) and low-to-intermediate pretest likelihood of CAD, a growing body of literature is showing that CCTA may have also a clinical role in patients with high pretest likelihood of CAD, known CAD and complex and diffuse CAD. Particularly, the SYNTAX studies demonstrated the usefulness of CCTA in the field of non-invasive assessment of these patients and planning of interventional and surgical coronary procedures, thanks to its ability to combine, in a single method, precise stenosis quantification, accurate plaque characterization, functional assessment and selection of the revascularization modality for any individual patient and of the vessels that need to be revascularized. Of note, the SYNTAX III Revolution trial showed, in patients with three-vessel CAD, that treatment decision-making between PCI and CABG based on CCTA only has an almost perfect agreement with the treatment decision derived from invasive coronary angiography (ICA). Moreover, the SYNTAX Score II demonstrated a high degree of correlation between the two diagnostic strategies, suggesting the potential feasibility of a treatment decision-making based solely on non-invasive imaging and clinical information. New research prospects have opened up for the future to demonstrate the true feasibility and safety of this innovative approach in the clinical arena.
\end{abstract}

Keywords: Cardiac CT; revascularization; syntax score

Submitted Sep 30, 2019. Accepted for publication Nov 20, 2019.

doi: $10.21037 / \mathrm{cdt} .2019 .11 .07$

View this article at: http://dx.doi.org/10.21037/cdt.2019.11.07

\section{Introduction}

Nearly fifteen years ago, some studies performed with old scanner generations such as 4- or 16-slice CT have shown that coronary CT angiography (CCTA) was a promising non-invasive tool for the detection of significant coronary stenoses. Particularly, the method has shown high sensitivity and excellent negative predictive value for ruling out obstructive coronary artery disease (CAD) (1). With the introduction in the clinical field of 64-slice CT between 2004 and 2006, CCTA started to be considered as 
an appropriate tool for the evaluation of coronary arteries in some specific clinical subsets. As reported by the European Society of Cardiology (2), the main clinical application of CCTA includes assessment of patients with stable chest pain and intermediate pretest likelihood of CAD (3).

During the last decade, an extensive literature confirmed the very high sensitivity, specificity and diagnostic accuracy of CCTA for the detection of coronary stenoses when compared to invasive coronary angiography (ICA) (4). Seven prospective multicenter studies showed the diagnostic accuracy of CCTA in patients with suspected but unknown CAD (5-10). Apart from the absolute value in diagnostic accuracy (in these trials sensitivity and specificity ranged between $85 \%$ and $99 \%$ and $64 \%$ and $92 \%$, respectively), the diagnostic performance of CCTA is influenced by the pretest likelihood of disease. Indeed, this imaging modality is associated with higher diagnostic accuracy mainly in patients with low-to-intermediate CAD risk (9), while a moderate specificity and positive predictive value has been found in high-risk patients (11). In them, coronary artery calcification (CAC) has been found to be the major underlying reason for disagreement between CCTA and quantitative ICA for the assessment of coronary lumen obstruction, with CCTA limited by a higher rate of overestimation but also by some clinically more dangerous cases of underestimation (12). However, technology advancements have recently shown to improve CCTA diagnostic accuracy, mainly in positive predictive value terms, in patients with high CAC and/or high pre-test likelihood of CAD. Indeed, scanners equipped with improved spatial resolution along the X-Y planes partially improved the positive predictive value in patients with high prevalence of obstructive CAD (13), while a study performed with dual-energy CT and calcium removal by material decomposition imaging demonstrated a significant increase in specificity and positive predictive value in patients with high CAC (14). When compared with other non-invasive tests (exercise ECG, stress echocardiography, SPECT or PET myocardial perfusion imaging and cardiac magnetic resonance), commonly used as gatekeepers to ICA in stable symptomatic patients, CCTA has demonstrated higher sensitivity and specificity versus exercise ECG (15), nuclear imaging (8) and all other stress imaging modalities $(8,10)$ in patients with intermediate pre-test probability and low prevalence of obstructive CAD (10). These results induced most of the people who drafted consensus documents, position papers and clinical guidelines to consider CCTA as a useful non-invasive tool in different clinical setting of patients with low-to-intermediate pre- test likelihood of CAD and low-to-moderate prevalence of obstructive CAD. However, the information provided by CCTA was still regarded insufficient to have a clinical role for the assessment of patients with diffuse and complex $\mathrm{CAD}$ and for planning interventional and surgical procedures of myocardial revascularization (16-18).

In order to overcome these intrinsic limitation, the group of Patrick Serruys evaluated in 2013 the feasibility and reproducibility of adapting to CCTA the SYNTAX score, a well-recognized ICA-based tool for stratifying CAD severity and complexity and for helping in the treatment decision-making in multivessel disease patients (19-24). Both European and US revascularization guidelines have adopted the anatomical SYNTAX score as an important tool for establishing the best revascularization strategy in patients with complex CAD (with or without unprotected left main coronary artery involvement) $(25,26)$. However, the absence of clinical variables in SYNTAX score calculation is a significant shortcoming of the method (27). Indeed, the guidelines state that clinical variables should also be taken into account during discussion of the multidisciplinary team consisting of a clinical cardiologist, a cardiac surgeon, and an interventional cardiologist (the so-called "heart team approach") when deciding for the best treatment modality. For this reason, a new score (SYNTAX score II) integrating six clinical variables (age, gender, creatinine clearance, peripheral vascular disease, COPD and left ventricular ejection fraction) has been developed. The addition of the patient clinical characteristics and comorbidities to the anatomical SYNTAX score giving rise to the SYNTAX Score II, provided a treatment recommendation based on the predicted 4-year mortality in patients undergoing coronary artery bypass grafting (CABG) surgery or percutaneous coronary intervention (PCI). Based on the SYNTAX Score II, in 2013 Serruys et al. created a dedicated nomogram that is able to provide a reliable and individualized prediction of 4-year mortality in patients undergoing CABG or PCI (27).

Technology advancements in the field of CCTA allowed expanding its use to patients with known CAD (28-32), thanks to a more accurate assessment of lumen narrowing and plaque burden $(13,33)$. However, despite the calculation of the CCTA-derived SYNTAX score has been shown to be accurate compared to the score resulting from ICA (19), the diagnostic performance of the latest CT scanner generation and the agreement on treatment decision in patients with multivessel CAD remain to be investigated. Therefore, the SYNTAX III Revolution trial tried to determine the 
agreement between separate heart teams on treatment recommendation based either on CCTA or ICA in patients with three-vessel CAD with or without left main disease (34).

\section{Clinical evidence in favor of CCTA use for planning myocardial revascularization}

The SYNTAX III Revolution trial was an international, multicenter study randomizing separate heart teams to make a treatment decision between CABG and PCI, using either CCTA or ICA, while blinded to the other imaging modality. Patients with three-vessel CAD with or without left main involvement but without prior revascularization who were diagnosed with either CCTA or ICA and were candidates for either CABG or PCI were assessed for eligibility (34). In addition to the initial imaging tool used for eligibility assessment, patients underwent evaluation with the alternative imaging modality. Separate heart teams constituted by an interventional cardiologist, a cardiac surgeon and a radiologist specialized in cardiac imaging were randomized to either assess the coronary anatomy with CCTA or ICA in addition to the patient clinical information. Each heart team calculated the anatomical SYNTAX score based solely on their allocated imaging modality and subsequently integrated the clinical information to compute the SYNTAX Score II risk prediction model providing a treatment recommendation, i.e., CABG, PCI, or equipoise between CABG and PCI. Patients underwent CCTA with one of the latest CT scanner generation, which has a nominal spatial resolution of 230 microns along the $\mathrm{X}-\mathrm{Y}$ planes, a rotational speed of $0.28 \mathrm{~s}$, and a Z-plane coverage of $16 \mathrm{~cm}$ enabling to image the heart in one heartbeat. Severity and extension of CAD were assessed using the anatomical SYNTAX score. Coronary segments showing narrowing with visual diameter stenosis $>50 \%$ using CCTA or ICA and weighted according to their location in the coronary tree were included in the calculation. For the primary endpoint, the two local heart teams made their decision on the choice of revascularization mode based on their clinical and anatomical assessment. For the secondary endpoint, fractional flow reserve derived from CCTA $\left(\mathrm{FFR}_{\mathrm{CT}}\right)$ was used to calculate the noninvasive functional SYNTAX score, which was computed subtracting non-flow limiting stenoses $\left(\mathrm{FFR}_{\mathrm{CT}}>0.80\right)$ from the CCTA-derived anatomical SYNTAX score (35). Finally, the non-invasive functional SYNTAX score was used to calculate the SYNTAX Score III, which conceptually is a combination of coronary anatomy complexity with its physiological repercussion and patient clinical characteristics and comorbidities. The diagnostic accuracy of $\mathrm{FFR}_{\mathrm{CT}}$, which is able to identify lesion-specific ischemia, has been validated in four prospective multicenter trials (36-38). In patients with multivessel disease, $\mathrm{FFR}_{\mathrm{CT}}$ has shown good diagnostic performance with invasive pressure-wire assessment as reference in a sub-analysis of the SYNTAX II trail (35). Moreover, the extent, severity and functional component of CAD can be objectively quantified using the functional SYNTAX score, which has a higher discrimination for clinical events compared to the anatomic SYNTAX score, while reducing interobserver variability (35). The main findings of the study (39) may be summarized as follows: (I) regarding the primary endpoint, the SYNTAX Score II provided a treatment recommendation of CABG in $28 \%$ of patients with CCTA and in $26 \%$ with ICA. The agreement concerning the recommendation on the type of revascularization between imaging modalities was high, as confirmed by the statistical nomenclature of Cohens' kappa showing a coefficient of 0.82 (95\% CI: 0.73-0.90). The mean difference between CCTA-derived and ICA-derived SYNTAX Score II was -0.01 (limits of agreement -5.1 to 4.9 ) with a correlation coefficient of $0.98(\mathrm{P}<0.001)$ without systematic or proportional differences. Moreover, CABG was selected as the revascularization strategy in 164 patients using CCTA and in 155 patients with ICA and the heart teams agreed on the coronary segments to be revascularized in $81.1 \%$ of the cases; (II) as regards the secondary endpoint, the study demonstrated that (40): (i) by including the noninvasive functional evaluation with $\mathrm{FFR}_{\mathrm{CT}}$, the heart teams changed treatment recommendation in $7 \%$ of the cases and modified the selection of vessels to be revascularized in $12 \%$ as compared to a CCTA assessment alone. Moreover, inclusion of $\mathrm{FFR}_{\mathrm{CT}}$ information on top of ICA changed treatment recommendation in $6.6 \%$ of the cases and modified revascularization planning in $18.3 \%$; (ii) the noninvasive functional SYNTAX score reclassified $15.5 \%$ of the patients to a lower SYNTAX score tertile based on CCTA and $14 \%$ of the patients to a lower SYNTAX score tertile based on ICA; (iii) in patients assessed by CCTA, FFR $_{\mathrm{CT}}$ reduced from 92.3 to 78.8 the percentage of patients with hemodynamically significant three-vessel CAD; finally, use of the non-invasive functional SYNTAX score for the SYNTAX Score II calculation (SYNTAX Score III) corrected the overestimation of the predicted mortality for PCI at 4 years (40). Of note, the acceptance rate for $\mathrm{FFR}_{\mathrm{CT}}$ analysis in the study was very high (88\%), 
particularly if compared with that reported in other prospective multicenter trials. For example, it was $33 \%$ in the PROMISE trial (41) and 69\% in the SYNTAX II (35). The high rate of CCTA suitability for $\mathrm{FFR}_{\mathrm{CT}}$ appears not surprising if we consider the scanner used in the SYNTAX III trial that combines different technical features, including the intracycle motion-correction algorithm, able to reducing the impact of motion artefacts on image quality (42). Particularly, this novel scanner combines a $0.23 \mathrm{~mm}$ spatial resolution, that may be effective in the reduction of beamhardening artefacts related to heavily calcific plaques, with a wide detector allowing $16-\mathrm{cm}$ of $\mathrm{Z}$-axis coverage and 0.28 seconds of gantry rotation time, allowing the acquisition in an axial scan mode of the whole cardiac volume in a single heart beat independently from the HR values, potentially avoiding motion artifacts due to high or irregular heart rate. Accordingly, recent phantom and human studies showed this scanner to be able to assess coronary arteries with excellent image quality and diagnostic accuracy up to HR of $100 \mathrm{bpm}$ during scanning $(43,44)$, including patients with rapid atrial fibrillation and HR-related small diastolic acquisition window $(42,45)$. However, because the presence of atrial fibrillation was an exclusion criterion of the SYNTAX III study, this unfavorable heart rhythm remains a potential limitation for the use of CCTA in patients with complex CAD. In summary, by randomizing two heart teams, the SYNTAX III study showed that treatment decision-making based on CCTA is in almost perfect agreement with the treatment decision derived from ICA in patients with three-vessel CAD. Moreover, the SYNTAX Score II demonstrated a high degree of correlation between the two diagnostic tools, suggesting the potential feasibility of treatment decision-making based solely on this type of non-invasive imaging modality and clinical information. For patients with multivessel disease, a multidisciplinary heart team approach is currently advocated by the guidelines for treatment decisionmaking with a Class I recommendation, level of evidence ' $\mathrm{C}$ ', in the absence of proof from randomized trial (46). The SYNTAX III REVOLUTION is the first trial to randomize the heart team and its almost perfect agreement upon treatment selection supports the usefulness of CCTA in patients with complex CAD. It should be also recognized that in the study the heart teams routinely included a radiologist in addition to an interventional cardiologist and a cardiac surgeon, which differs from the definition of a heart team given by the guidelines. Undoubtedly, the interaction with the radiologist enhanced image interpretation and the decision-making process.

Although these findings are encouraging, some concerns remain on CCTA capability to be used for decision-making in patients with a high calcific burden of the coronary arteries, a frequent condition in complex and diffuse $\mathrm{CAD}$, particularly in elderly and diabetic patients. Indeed, CCTA images are less accurate and interpretable in these settings, often leading to overestimation of lesion severity with a negative impact on specificity and accuracy of the method $(1,47)$. This issue was addressed by a sub-analysis of the SYNTAX III trial showing, as expected, that heavy coronary calcifications moderately affect CCTA capability to accurately assess the anatomical SYNTAX score, with a significantly higher difference between the CCTA-derived and ICA-derived anatomical SYNTAX score (difference of 5.9 points in heavy calcification patients $v s .1 .5$ points in patients without heavy calcifications, respectively, $\mathrm{P}=0.004$ ). However, despite the discrepancy in the anatomical SYNTAX score assessment, agreement on the heart team treatment decision did not differ in patients with (Cohen's Kappa 0.79) or without heavy calcifications (Cohen's Kappa 0.84). Similarly, agreement on treatment planning, defined as the coronary vessels to be revascularized, was high and did not differ between patients with (overall vessels concordance $80.3 \%$ ) or without heavy calcifications (overall vessels concordance 82.8\%) (48). Despite these findings it is important to note that use of CCTA in patients with complex CAD remains a promising new scenario for this non-invasive modality but lacks of widespread acceptance. Indeed, although the recent ESC Guidelines on chronic coronary syndrome promoted CCTA in Class I of recommendation in patients with suspected CAD, the method is still considered as the most appropriate in patients with low-to intermediate pretest likelihood of CAD, whereas imaging stress tests (echo, CMR, SPECT, PET) are preferable in patients at higher risk (49).

\section{Clinical cases}

We report two case examples (Figures 1-5) in which CCTA together with $\mathrm{FFR}_{\mathrm{CT}}$ was used successfully for planning PCI. The case shown in Figures 1-3 illustrates the high diagnostic accuracy of $\mathrm{FFR}_{\mathrm{CT}}$ in a patient with multivessel CAD, confirming the findings of Collet et al. in a subanalysis of the SYNTAX II trial (35). The case presented in Figures 4,5 shows how the new dedicated CCTA software developed for plaque characterization and quantification is able to predict IVUS findings, as demonstrated by a recent 

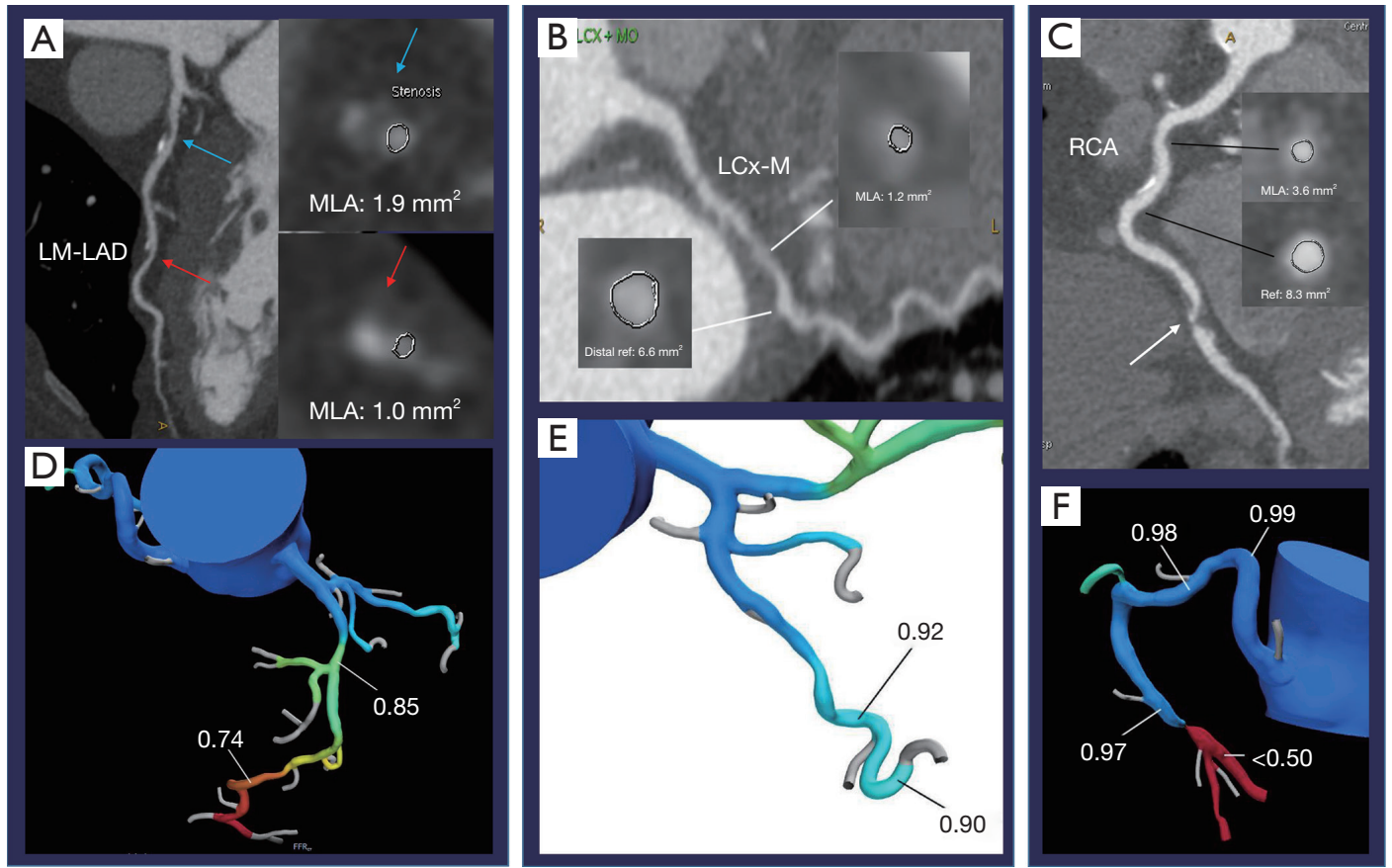

Figure 1 CCTA curved multiplanar reconstructions show the presence of two $>50 \%$ stenoses of the proximal (arrow) and mid (arrow) LAD (A), a long stenosis of the proximal LCx (B) and two stenoses in the mid (>50\%) and distal part (tight lesion, arrow) of the RCA (C). The $\mathrm{FFR}_{\mathrm{CT}}$ assessment confirmed the functional significance of the stenosis in mid LAD (D) and distal RCA (F), whereas the FFR $\mathrm{CT}_{\mathrm{value}}$ was $>0.80$ after the lesions in proximal LAD (D), LCx (E) and proximal RCA (F). CCTA, coronary CT angiography; LM, left main coronary artery; LAD, left anterior descending artery; LCx, left circumflex artery; M, marginal branch; RCA, right coronary artery.

study of Conte et al. (33).

\section{Future directions}

The SYNTAX III Revolution study opens new perspectives on CCTA use as a tool to provide interventionalists and cardiac surgeons with an anatomy and functional noninvasive road-map for planning myocardial revascularization strategies. Moreover, future full automation of the SYNTAX III score calculation has the potential to further enhance and speed up the decision-making process in patients with multivessel disease. The interactive planner, which is a new application of $\mathrm{FFR}_{\mathrm{CT}}$, may improve treatment selection while tailoring procedural strategy based on assessment of functional outcome after virtual treatment (50). It is relevant to note that, although a prototype of the algorithm applied to coronary stents suggested a potential usefulness in this clinical field (51), the FFR $_{\text {CT }}$ calculation is still not validated and available for the routine clinical use in patients with stented coronary arteries. Although this limitation, a CCTA/FFR ${ }_{\mathrm{CT}}$ diagnostic work- up has been demonstrated to be cost-effective in patients with suspected CAD and without history of previous coronary revascularization. In the PLATFORM study, at one-year follow-up of patients in the planned invasive test group, $\mathrm{FFR}_{\mathrm{CT}}$ guided strategy cost was $\$ 8,127$ vs. $\$ 12,145$ with a usual care strategy $(\mathrm{P}<0.0001)$, not accounting for the cost of the $\mathrm{FFR}_{\mathrm{CT}}$ test. The mean costs remained $26 \%$ lower among the $\mathrm{FFR}_{\mathrm{CT}}$ patients than among usual care patients $(\$ 9,036$ vs. $\$ 12,145, \mathrm{P}<0.0001)$ when factoring in the cost of the $\mathrm{FFR}_{\mathrm{CT}}$ analysis (52). At the present time, the major insurance companies in Europe and US recognize the reimbursement for $\mathrm{FFR}_{\mathrm{CT}}$ analysis. However, the $\mathrm{FFR}_{\mathrm{CT}}$ software is proprietary and still not widely available. In this novel and growing clinical field, stress myocardial CT perfusion (CTP) has been introduced as a new tool for evaluating the functional relevance of a coronary stenosis (53-57). In the field of complex CAD treated with PCI, the ADVANTAGE prospective study recently evaluated 150 patients with previous coronary stent implantation and demonstrated a CTP diagnostic accuracy significantly higher than that of CCTA in the territory-based and patient- 
Anatomical and Functional CCTA-derived SYNTAX Score
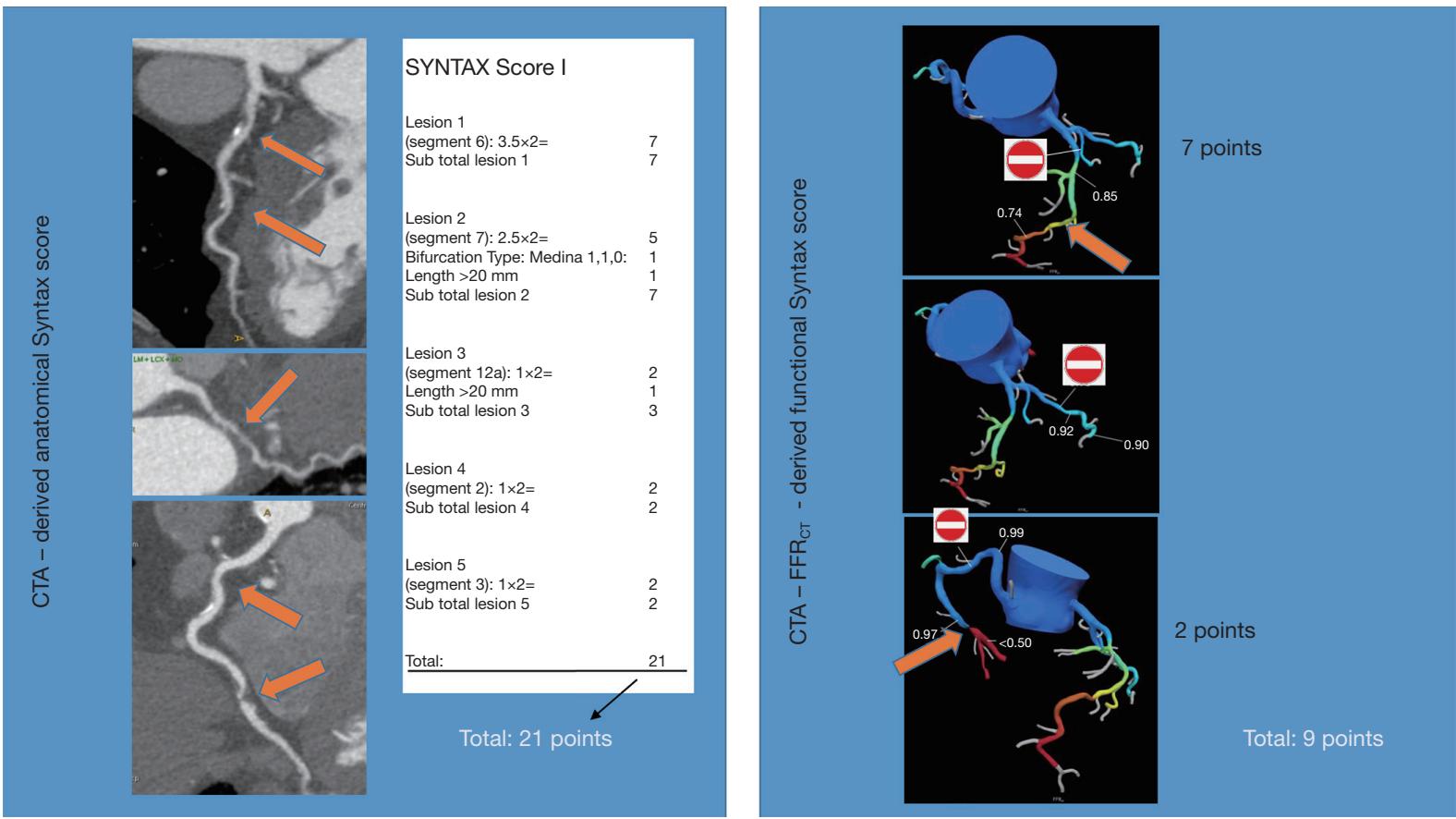

Figure 2 Anatomical (left panel) and functional (right panel) CCTA-derived SYNTAX Score calculations. The anatomical Syntax score of 21, derived by the scoring of five lesions (proximal-mid LAD, long lesion in LCx, mid-distal RCA, arrows) was downgraded to 9 points only after the incorporation of $\mathrm{FFR}_{\mathrm{CT}}$ into the functional Syntax score, by the exclusion of proximal LAD (arrow), LCx and proximal RCA (arrow) stenoses. The reduced value of the functional Syntax score suggested PCI instead of CABG. LM, left main coronary artery; LAD, left anterior descending artery; LCx, left circumflex artery; M, marginal branch; RCA, right coronary artery.
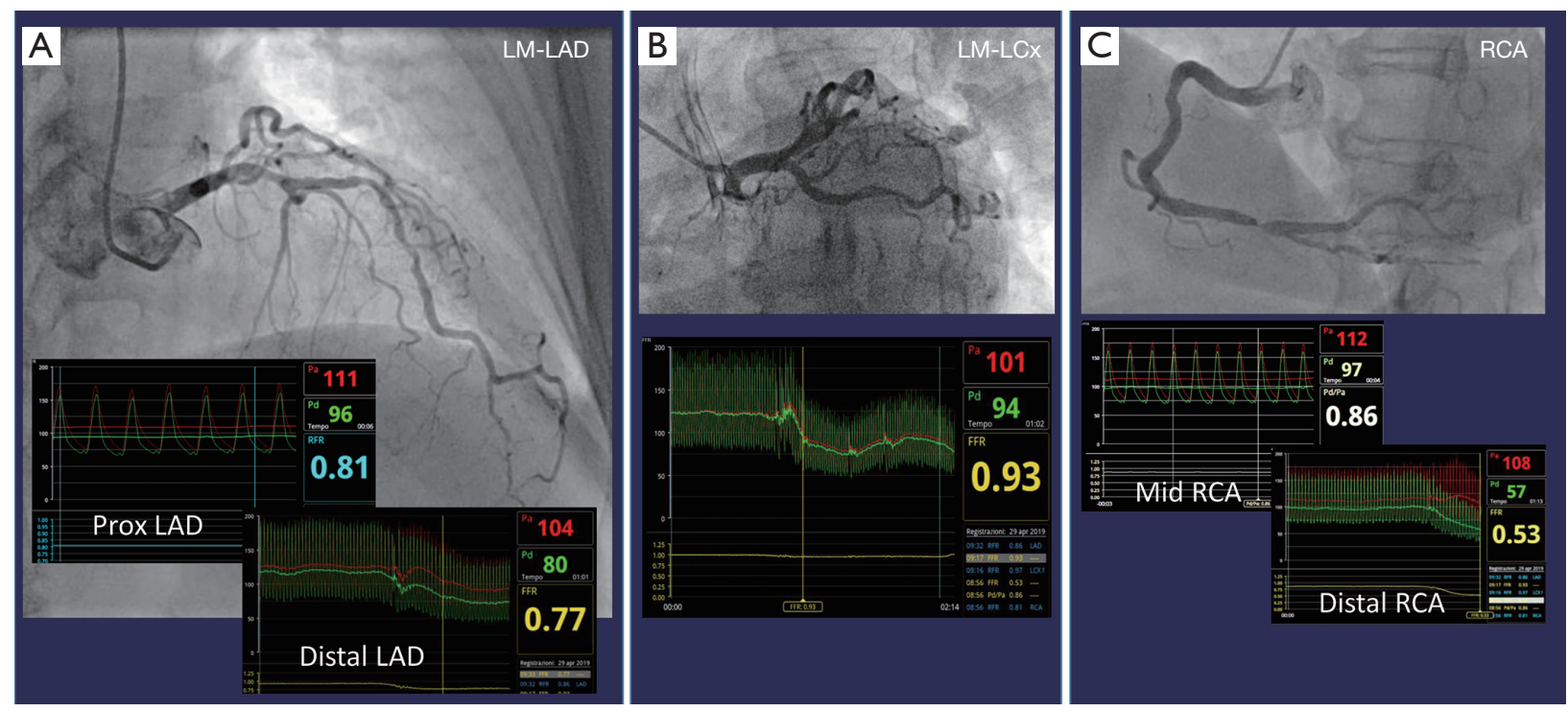

Figure 3 Invasive coronary angiography and invasive FFR assessment of LM-LAD (A), LCx-M branch (B) and RCA (C) show a very good agreement of both anatomical and functional invasive assessment with the correspondent non-invasive evaluation by CCTA- FFR ${ }_{\mathrm{CT}}$. $\mathrm{LM}_{\text {, }}$ left main coronary artery; LAD, left anterior descending artery; LCx, left circumflex artery; M, marginal branch; RCA, right coronary artery. 

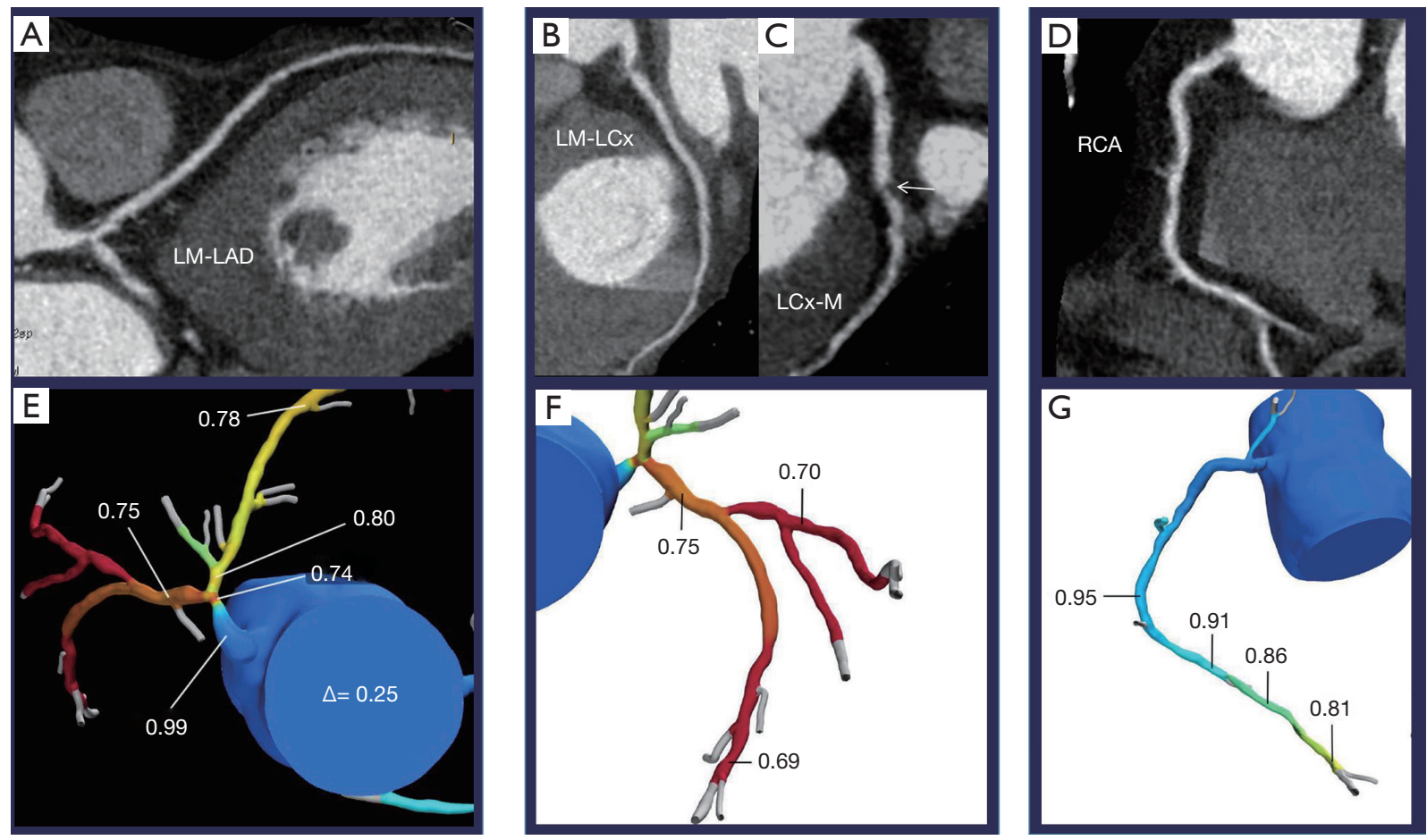

Figure 4 CCTA curved multiplanar reconstructions show a $70 \%$ stenosis of the distal left main coronary artery (A), involving the ostium of LCx (B) and a tight stenosis at the ostium of obtuse marginal branch (C, arrow), whereas the RCA was free from >50\% stenosis (D). The $\mathrm{FFR}_{\mathrm{CT}}$ assessment confirmed the functional significance of the stenosis in distal LM-ostial LCx (E) and obtuse marginal branch (F), whereas the $\mathrm{FFR}_{\mathrm{CT}}$ values of the RCA were above the ischemia threshold of $0.80(\mathrm{G})$. LM, left main coronary artery; LAD, left anterior descending artery; LCx, left circumflex artery; $\mathrm{M}$, marginal branch; RCA, right coronary artery.

based analyses $(92.1 \%$ vs. $85.6 \%$ and $86.7 \%$ vs. $76.7 \%$, respectively), using QCA as gold standard. Similarly, CTP specificity and diagnostic accuracy were significantly higher than those of CCTA when invasive FFR was employed as gold standard. Of note, the radiation exposure of cardiac CT (CCTA + CTP) was $4.15 \pm 1.5 \mathrm{mSv}$ (32) To the best of our knowledge, only few studies addressed the prognostic role and the cost-effectiveness of CTP. In 2017, Meinel et al. enrolled 144 patients who underwent both CCTA and dynamic CTP. The study showed that CTP had incremental predictive value over clinical risk factors and detection of CAD with CCTA (58). More

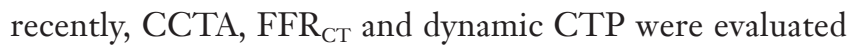
in a multicenter trial that included 84 patients. The trial demonstrated that myocardial blood flow evaluated by dynamic CTP has the highest prognostic value, over CCTA and $\mathrm{FFR}_{\mathrm{CT}}$, in terms of future major cardiac events at 18-month follow-up (59).

The SYNTAX III study raises the question if a cardiac surgeon might be confident in using only the non-invasive coronary road-map provided by CCTA to plan CABG. The intriguing hypothesis was tested first in a theoretical feasibility survey study (60), in which six surgeons of the SYNTAX III Revolution trial were invited to review CCTA scans of 20 patients who previously underwent CABG during the course of the trial. Each surgeon had to declare whether planning and execution of surgery would be feasible and safe with the sole anatomical and functional assessment of CCTA as procedural guidance. The results of this "live survey" were quite impressive. The opinion of the surgeons was that $84 \%$ of the cases were eligible for surgery without ICA evaluation. Based on these findings, a "first-in-man", proof-of-concept feasibility and safety trial has been designed and will enroll 100 patients in whom surgeons will perform CABG without having access to ICA. Of note, CABG outcome will be assessed by CCTA 30 days after surgery in order to evaluate graft patency and correct anatomic location of the anastomoses (61). 


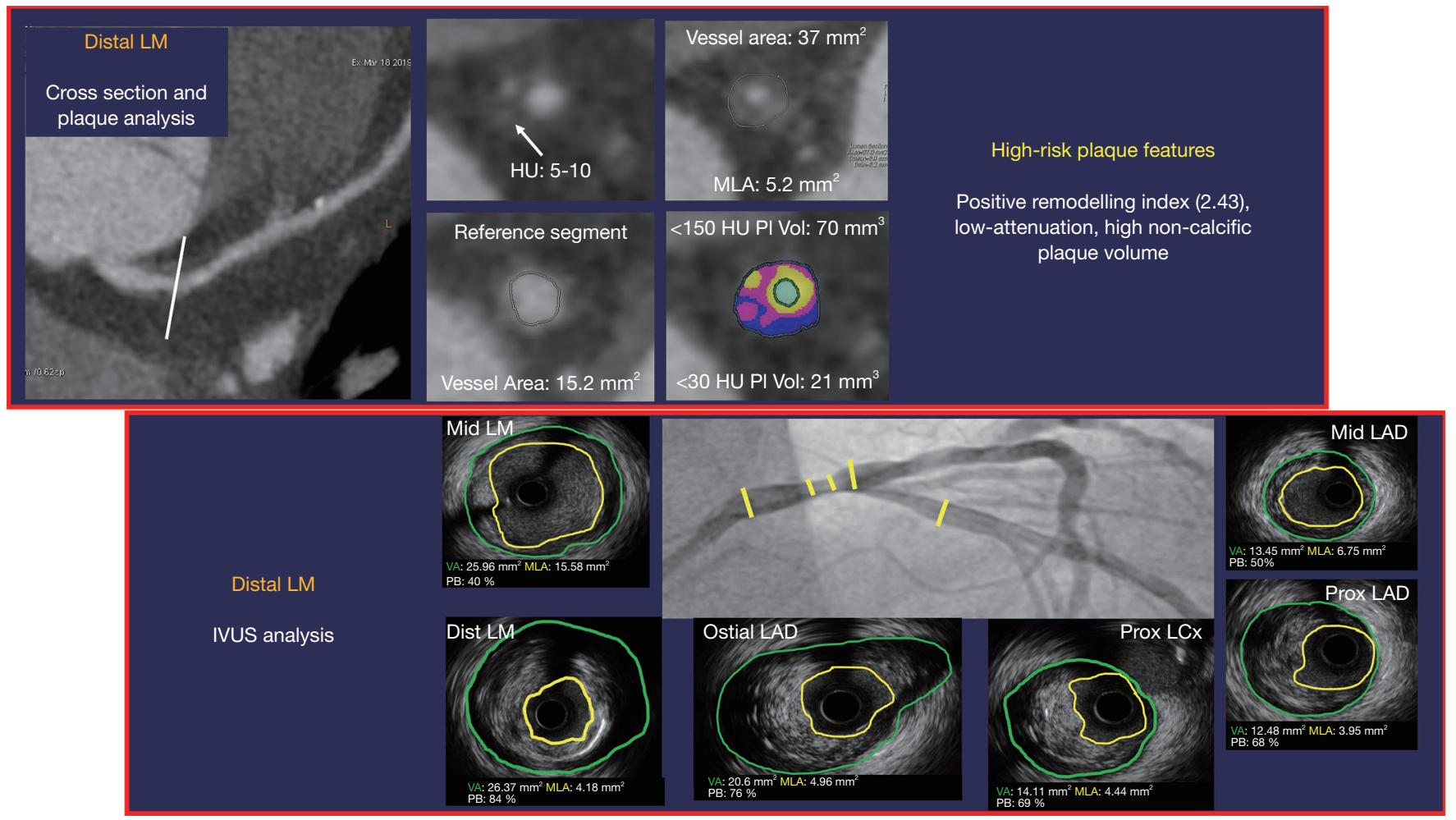

Figure 5 CCTA advanced plaque analysis by a dedicated software at the level of distal LM-ostial LAD (upper panel), showing the presence of qualitative (positive remodeling, low attenuation plaque) (arrow) and quantitative [high non-calcific (HU < 150) plaque volume] high-risk parameters. IVUS assessment (lower panel) shows similar values of plaque volume and minimal lumen area (MLA) in comparison with those measured with CCTA (MLA at distal LM 5.2 vs. $4.96 \mathrm{~mm}^{2}$, CCTA vs. IVUS, respectively). IVUS, intravascular ultrasound; MLA, minimal lumen area.

\section{Conclusions}

An emerging body of literature indicates that CCTA may have a clinical role also in patients with high pretest likelihood of CAD, known CAD and complex and diffuse disease. In particular, the SYNTAX studies demonstrated the usefulness of CCTA as a non-invasive tool for planning interventional and surgical coronary procedures, thanks to its ability to combine, in a single method, precise stenosis quantification, accurate plaque characterization, functional assessment and selection of the revascularization modality for any individual patient and of the vessels that need to be revascularized. It may be reasonable to state that the time has come to use CCTA for the assessment and decision making of patients with multivessel CAD (62).

\section{Acknowledgments}

Funding: None.

\section{Footnote}

Provenance and Peer Review: This article was commissioned by the Guest Editor (Filippo Cademartiri) for the series "Clinical Impact of Cardiac CT in Clinical Practice" published in Cardiovascular Diagnosis and Therapy. The article was sent for external peer review organized by the Guest Editor and the editorial office.

Conflicts of Interest: All authors have completed the ICMJE uniform disclosure form (available at http://dx.doi. org/10.21037/cdt.2019.11.07). The series "Impact of Cardiac CT in Clinical Practice" was commissioned by the editorial office without any funding or sponsorship. Dr. PWS reports grants from Heartflow, grants from General Electronic Healthcare, during the conduct of the study; personal fees from Philips/Volcano, personal fees from Xeltis, personal fees from Sino Medical Sciences, outside the submitted work. The other authors have no other conflicts of interest 
to declare.

Ethical Statement: The authors are accountable for all aspects of the work in ensuring that questions related to the accuracy or integrity of any part of the work are appropriately investigated and resolved.

Open Access Statement: This is an Open Access article distributed in accordance with the Creative Commons Attribution-NonCommercial-NoDerivs 4.0 International License (CC BY-NC-ND 4.0), which permits the noncommercial replication and distribution of the article with the strict proviso that no changes or edits are made and the original work is properly cited (including links to both the formal publication through the relevant DOI and the license). See: https://creativecommons.org/licenses/by-nc-nd/4.0/.

\section{References}

1. Stein PD, Yaekoub AY, Matta F, et al. 64-slice CT for diagnosis of coronary artery disease: a systematic review. Am J Med 2008;121:715-25.

2. Schroeder S, Achenbach S, Bengel F, et al. Cardiac computed tomography: indications, applications, limitations, and training requirements: report of a Writing Group deployed by the Working Group Nuclear Cardiology and Cardiac CT of the European Society of Cardiology and the European Council of Nuclear Cardiology. Eur Heart J 2008;29:531-56.

3. Budoff MJ, Dowe D, Jollis JG, et al. Diagnostic Performance of 64-Multidetector Row Coronary Computed Tomographic Angiography for Evaluation of Coronary Artery Stenosis in Individuals Without Known Coronary Artery Disease. J Am Coll Cardiol 2008;52:1724-32.

4. Menke J, Kowalski J. Diagnostic accuracy and utility of coronary CT angiography with consideration of unevaluable results: A systematic review and multivariate Bayesian random-effects meta-analysis with intention to diagnose. Eur Radiol 2016;26:451-8.

5. Meijboom WB, Meijs MF, Schuijf JD, et al. Diagnostic accuracy of 64-slice computed tomography coronary angiography: a prospective, multicenter, multivendor study. J Am Coll Cardiol 2008;52:2135-44.

6. Marano R, De Cobelli F, Floriani I, et al. Italian multicenter, prospective study to evaluate the negative predictive value of 16- and 64-slice MDCT imaging in patients scheduled for coronary angiography
(NIMISCAD-Non Invasive Multicenter Italian Study for Coronary Artery Disease). Eur Radiol 2009;19:1114-23.

7. Budoff MJ, Kalia N, Cole J, et al. Diagnostic accuracy of Visipaque enhanced coronary computed tomographic angiography: a prospective multicenter trial. Coron Artery Dis 2017;28:52-6.

8. Budoff MJ, Li D, Kazerooni EA, et al. Diagnostic Accuracy of Noninvasive 64-row Computed Tomographic Coronary Angiography (CCTA) Compared with Myocardial Perfusion Imaging (MPI): The PICTURE Study, A Prospective Multicenter Trial. Acad Radiol 2017;24:22-9.

9. Arbab-Zadeh A, Miller J, Rochitte C, et al. Diagnostic Accuracy of Computed Tomography Coronary Angiography According to Pre-Test Probability of Coronary Artery Disease and Severity of Coronary Arterial Calcification. The CORE-64 (Coronary Artery Evaluation Using 64-Row Multidetector Computed Tomography Angiography) International Multicenter Study. J Am Coll Cardiol 2012;59:379-87.

10. Neglia D, Rovai D, Caselli C, et al. Detection of significant coronary artery disease by noninvasive anatomical and functional imaging. Circ Cardiovasc Imaging 2015;8:e002179.

11. Pontone G, Andreini D, Quaglia C, et al. Accuracy of multidetector spiral computed tomography in detecting significant coronary stenosis in patient populations with differing pre-test probabilities of disease. Clin Radiol 2007;62:978-85.

12. Vavere AL, Arbab-Zadeh A, Rochitte CE, et al. Coronary artery stenoses: accuracy of 64-detector row CT angiography in segments with mild, moderate, or severe calcification--a subanalysis of the CORE-64 trial. Radiology 2011;261:100-8.

13. Pontone G, Bertella E, Mushtaq S, et al. Coronary artery disease: diagnostic accuracy of CT coronary angiography-a comparison of high and standard spatial resolution scanning. Radiology 2014;271:688-94.

14. Andreini D, Pontone G, Mushtaq S, et al. Diagnostic Accuracy of Rapid Kilovolt Peak-Switching Dual-Energy CT Coronary Angiography in Patients With a High Calcium Score. JACC Cardiovasc Imaging 2015;8:746-8.

15. Dedic A, Genders TS, Ferket BS, et al. Stable angina pectoris: head-to- head comparison of prognostic value of cardiac CT and exercise testing. Radiology 2011;261:42836.e39.

16. Task Force Members; Montalescot G, Sechtem U, et al. 2013 ESC guidelines on the management of stable coronary artery disease: the Task Force on the 
management of stable coronary artery disease of the European Society of Cardiology. Practice Guideline Eur Heart J 2013;34:2949-3003.

17. Wolk MJ, Bailey SR, Doherty JU, et al. ACCF/AHA/ ASE/ASNC/HFSA/HRS/SCAI/SCCT/SCMR/STS 2013 multimodality appropriate use criteria for the detection and risk assessment of stable ischemic heart disease. American College of Cardiology Foundation Appropriate Use Criteria Task Force. J Am Coll Cardiol 2014;63:380-406.

18. Andreini D, Martuscelli E, Guaricci AI, et al. Clinical recommendations on Cardiac-CT in 2015: a position paper of the Working Group on Cardiac-CT and Nuclear Cardiology of the Italian Society of Cardiology. Working Group on Cardiac-CT and Nuclear Cardiology of the Italian Society of Cardiology. J Cardiovasc Med (Hagerstown) 2016;17:73-84.

19. Papadopoulou SL, Girasis C, Dharampal A, et al. CTSYNTAX score: a feasibility and reproducibility study. JACC Cardiovasc Imaging 2013;6:413-5.

20. Serruys PW, Morice MC, Kappetein AP, et al.

Percutaneous coronary intervention versus coronary-artery bypass grafting for severe coronary artery disease. N Engl J Med 2009;360:961-72.

21. Serruys PW, Onuma Y, Garg S, et al. Assessment of the SYNTAX score in the Syntax study. EuroIntervention 2009;5:50-6.

22. Sianos G, Morel MA, Kappetein AP, et al. The SYNTAX Score: an angiographic tool grading the complexity of coronary artery disease. EuroIntervention 2005;1:219-27.

23. Morice MC, Serruys PW, Kappetein AP, et al. Outcomes in patients with de novo left main disease treated with either percutaneous coronary intervention using paclitaxeleluting stents or coronary artery bypass graft treatment in the Synergy Between Percutaneous Coronary Intervention with TAXUS and Cardiac Surgery (SYNTAX) trial. Circulation 2010;121:2645-53

24. Ong AT, Serruys PW, Mohr FW, et al. The SYNergy between percutaneous coronary intervention with TAXus and cardiac surgery (SYNTAX) study: design, rationale, and run-in phase. Am Heart J 2006;151:1194-204.

25. Wijns W, Kolh P, Danchin N, et al. Guidelines on myocardial revascularization: the Task Force on Myocardial Revascularization of the European Society of Cardiology (ESC) and the European Association for Cardio-Thoracic Surgery (EACTS). Eur Heart J 2010;31:2501-55.

26. Levine GN, Bates ER, Blankenship JC, et al. 2011 ACCF/AHA/SCAI Guideline for Percutaneous Coronary Intervention. A report of the American College of
Cardiology Foundation/American Heart Association Task Force on Practice Guidelines and the Society for Cardiovascular Angiography and Interventions. J Am Coll Cardiol 2011;58:e44-122.

27. Farooq V, van Klaveren D, Steyerberg EW, et al. Anatomical and clinical characteristics to guide decision making between coronary artery bypass surgery and percutaneous coronary intervention for individual patients: development and validation of SYNTAX score II. Lancet 2013;381:639-50.

28. Mushtaq S, Conte E, Pontone G, et al. Interpretability of coronary CT angiography performed with a novel whole-heart coverage high-definition CT scanner in 300 consecutive patients with coronary artery bypass grafts. J Cardiovasc Comput Tomogr 2020;14:137-43.

29. Andreini D, Pontone G, Mushtaq S, Coronary in-stent restenosis: assessment with CT coronary angiography. Radiology 2012;265:410-7.

30. Mushtaq S, Andreini D, Pontone G, et al. Prognostic value of coronary CTA in coronary bypass patients: a long-term follow-up study. JACC Cardiovasc Imaging 2014;7:580-9.

31. Andreini D, Pontone G, Mushtaq S, et al. Diagnostic performance of two types of low radiation exposure protocol for prospective ECG-triggering multidetector computed tomography angiography in assessment of coronary artery bypass graft. Int J Cardiol 2012;157:63-9.

32. Andreini D, Mushtaq S, Pontone G, et al. CT Perfusion Versus Coronary CT Angiography in Patients With Suspected In-Stent Restenosis or CAD Progression. JACC Cardiovasc Imaging 2020;13:732-42.

33. Conte E, Mushtaq S, Pontone G, et al. Plaque quantification by coronary computed tomography angiography using intravascular ultrasound as a reference standard: a comparison between standard and last generation computed tomography scanners. Eur Heart J Cardiovasc Imaging 2020;21:191-201.

34. Cavalcante R, Onuma Y, Sotomi Y, et al. Non-invasive Heart Team assessment of multivessel coronary disease with coronary computed tomography angiography based on SYNTAX score II treatment recommendations: design and rationale of the randomised SYNTAX III. Revolution trial. EuroIntervention 2017;12:2001-8.

35. Collet C, Miyazaki Y, Ryan N, et al. Fractional flow reserve derived from computed tomographic angiography in patients with multivessel CAD. J Am Coll Cardiol 2018;71:2756-69.

36. Min JK, Leipsic J, Pencina MJ, et al. Diagnostic accuracy of fractional flow reserve from anatomic CT angiography. 
JAMA 2012;308:1237-45.

37. Koo BK, Erglis A, Doh JH, et al. Diagnosis of ischemia-causing coronary stenoses by noninvasive fractional flow reserve computed from coronary computed tomographic angiograms. Results from the prospective multicenter DISCOVER-FLOW (Diagnosis of Ischemia-Causing Stenoses Obtained Via Noninvasive Fractional Flow Reserve) study. J Am Coll Cardiol 2011;58:1989-97.

38. Norgaard BL, Leipsic J, Gaur S, et al. Diagnostic performance of noninvasive fractional flow reserve derived from coronary computed tomography angiography in suspected coronary artery disease: the NXT trial (Analysis of Coronary Blood Flow Using CT Angiography: Next Steps). J Am Coll Cardiol 2014;63:1145-55.

39. Collet C, Onuma Y, Andreini D, et al. Coronary Computed Tomography Angiography for Heart Team Decision-making in Multivessel Coronary Artery Disease. Eur Heart J 2018;39:3689-98.

40. Andreini D, Modolo R, Katagiri Y, et al. Impact of Fractional Flow Reserve Derived from Coronary Computed Tomography Angiography on Heart Team Treatment Decision-Making in Patients with Multivessel Coronary Artery Disease: Insights from the Syntax III Revolution trial. Circ Cardiovasc Interv 2019;12:e007607.

41. Lu MT, Ferencik M, Roberts RS, et al. Noninvasive FFR Derived From Coronary CT Angiography: Management and Outcomes in the PROMISE Trial. JACC Cardiovasc Imaging 2017;10:1350-8.

42. Andreini D, Pontone G, Mushtaq S, et al. Image quality and radiation dose of coronary CT angiography performed with whole-heart coverage CT scanner with intracycle motion correction algorithm in patients with atrial fibrillation. Eur Radiol 2018;28:1383-92.

43. Cho I, Elmore K, Hartaigh BÓ, et al. Heart-rate dependent improvement in image quality and diagnostic accuracy of coronary computed tomographic angiography by novel intracycle motion correction algorithm, Clin Imaging 2015;39:421-6.

44. Andreini D, Mushtaq S, Pontone G, et al. Diagnostic performance of coronary CT angiography carried out with a novel whole-heart coverage high-definition CT scanner in patients with high heart rate. Int J Cardiol 2018;257:325-31.

45. Andreini D, Pontone G, Mushtaq S, et al. Atrial fibrillation: diagnostic accuracy of coronary CT angiography performed with a whole-heart 230-mm spatial resolution CT scanner. Radiology 2017;284:676-84.
46. Windecker S, Kolh P, Alfonso F, et al. 2014 ESC/EACTS Guidelines on myocardial revascularization: the task force on myocardial revascularization of the European Society of Cardiology (ESC) and the European Association for Cardio-Thoracic Surgery (EACTS) Developed with the special contribution of the European Association of Percutaneous Cardiovascular Interventions (EAPCI). Eur Heart J 2014;35:2541-619.

47. Andreini D, Pontone G, Bartorelli AL, et al. Comparison of the diagnostic performance of 64-slice computed tomography coronary angiography in diabetic and nondiabetic patients with suspected coronary artery disease. Cardiovasc Diabetol 2010;9:80.

48. Andreini D, Takahashi K, Mushtaq S, et al. Impact of Coronary Calcification on the Agreement between Coronary Computed Tomography Angiography CTA and Invasive Coronary Conventional Angiography in the SYNTAX Score Treatment Decision: Insights from the Syntax III Revolution trial. J Am Coll Cardiol 2018;72:TCT-674.

49. Task Force Members. 2019 ESC Guidelines for the diagnosis and management of chronic coronary syndromes The Task Force for the diagnosis and management of chronic coronary syndromes of the European Society of Cardiology (ESC).

50. Sonck J, Miyazaki Y, Mandry D, et al. Non-invasive planning of complex coronary artery disease using a novel Interactive Planner for PCI. EuroIntervention 2017. [Epub ahead of print].

51. Andreini D, Mushtaq S, Pontone G, et al. Severe instent restenosis missed by coronary CT angiography and accurately detected with FFRCT. Int J Cardiovasc Imaging 2017;33:119-20.

52. Hlatky MA, De Bruyne B, Pontone G, et al. Quality-ofLife and Economic Outcomes of Assessing Fractional Flow Reserve With Computed Tomography Angiography: PLATFORM. J Am Coll Cardiol 2015;66:2315-23.

53. Rochitte CE, George RT, Chen MY, et al. Computed tomography angiography and perfusion to assess coronary artery stenosis causing perfusion defects by single photon emission computed tomography: the CORE320 study. Eur Heart J 2014;35:1120-30.

54. George RT, Arbab-Zadeh A, Miller JM, et al. Computed tomography myocardial perfusion imaging with 320row detector computed tomography accurately detects myocardial ischemia in patients with obstructive coronary artery disease. Circ Cardiovasc Imaging 2012;5:e333-e340.

55. Osawa K, Miyoshi T, Koyama Y, et al. Additional 
diagnostic value of first-pass myocardial perfusion imaging without stress when combined with 64-row detector coronary CT angiography in patients with coronary artery disease. Heart 2014;100:1008-15.

56. Greif M, von Ziegler F, Bamberg F, et al. CT stress perfusion imaging for detection of haemodynamically relevant coronary stenosis as defined by FFR. Heart 2013;99:1004-11.

57. Pontone G, Andreini D, Guaricci AI, et al. Incremental Diagnostic Value of Stress Computed Tomography Myocardial Perfusion With Whole-Heart Coverage CT Scanner in Intermediate- to High-Risk Symptomatic Patients Suspected of Coronary Artery Disease. JACC Cardiovasc Imaging 2019;12:338-49.

58. Meinel FG, Pugliese F, Schoepf UJ, et al. Prognostic value of stress dynamic myocardial perfusion CT in a multicenter population with known or suspected coronary artery disease. AJR Am J Roentgenol 2017;208:761-9.

59. van Assen M, De Cecco CN, Eid M, et al. Prognostic

Cite this article as: Andreini D, Mushtaq S, Conte E, Mei M, Nicoli F, Melotti E, Pompilio G, Pepi M, Bartorelli AL, Onuma Y, Serruys PW. The usefulness of cardiac CT integrated with FFRCT for planning myocardial revascularization in complex coronary artery disease: a lesson from SYNTAX studies. Cardiovasc Diagn Ther 2020;10(6):2036-2047. doi: 10.21037/ cdt.2019.11.07 value of CT myocardial perfusion imaging and CT-derived fractional flow reserve for major adverse cardiac events in patients with coronary artery disease. J Cardiovasc Comput Tomogr 2019;13:26-33.

60. Sonck J, Miyazaki Y, Collet C, et al. Feasibility of planning coronary artery bypass grafting based only on coronary computed tomography angiography and CT-derived fractional flow reserve: a pilot survey of the surgeons involved in the randomized SYNTAX III Revolution trial. Interact Cardiovasc Thorac Surg 2019. [Epub ahead of print].

61. Serruys PW, Chichareon P, Modolo R, et al. The SYNTAX score on its way out or ... towards artificial intelligence: part II. EuroIntervention 2020;16:60-75.

62. Andreini D, Onuma Y, Bartorelli AL, et al. The time has come to use coronary CT angiography in patients with multivessel coronary artery disease. Eur Heart J 2019;40:1472. 